\title{
The effects of particle size, fermentation and roasting of cocoa nibs on supercritical fluid extraction of cocoa butter
}

\begin{abstract}
The effects of particle size, fermentation, roasting time and roasting temperature of cocoa nibs on cocoa butter extraction using supercritical fluid technology were studied. The effect of particle size was studied using cocoa liquor $(\mathrm{D}=0.074 \mathrm{~mm})$, ground cocoa nib $(\mathrm{D}=0.25-$ $0.50 \mathrm{~mm}$ and $1.0-1.2 \mathrm{~mm}$ ) and whole cocoa nibs at $35 \mathrm{MPa}, 60{ }^{\circ} \mathrm{C}$ and flow rate of $2 \mathrm{ml} / \mathrm{min}$ using supercritical carbon dioxide (SC-CO2). The effect of degree of fermentation was studied using unfermented, partly fermented and fermented cocoa, whereas the effect of roasting using roasted and unroasted cocoa nibs. Fermentation and roasting studies were conducted under the same operation conditions as particle size study using SC-CO2 but with ethanol $(25 \% \mathrm{w} / \mathrm{w})$ as cosolvent. Cocoa butter extracted from the three studies was analyzed for total fat content (\%), triglycerides and fatty acid methyl ester. The results showed that the extraction yield was significantly increased by a reduction in particle size. The highest yield was also obtained using unfermented cocoa, roasted for $35 \mathrm{~min}$ and at $150{ }^{\circ} \mathrm{C}$. Generally, cocoa butter had similar triglycerides and fatty acid methyl ester composition at 5, 10 and 15 $\mathrm{h}$ extraction time. Glycerol-1,3-dipalmitate-2-oleate (POP), glycerol-1-palmitate-2-oleate-3stearate (POS), and glycerol-1,3-distearate-2-oleate (SOS) account for most of the triglycerides, with POS (42.52-46.44\%) being the major component. Palmitic, stearic and oleic were the main fatty acids in the extracted cocoa butter, with stearic acid being the highest component (33.70-40.22\%).
\end{abstract}

Keyword: Cocoa butter, Particle size, Fermentation, Roasting, Supercritical fluid extraction 